\title{
Estrategias de enseñanza para la integración del conocimiento autodidacta y académico en Educación Musical
}

\section{Teaching strategies for the integration of self-taught and academic knowledge in Music Education}

\author{
Sergio Linares Blanco \\ profesorslb@gmail.com \\ Código ORCID: 0000-0003-0339-4378 \\ Universidad Pedagógica Experimental Libertador, Venezuela
}

RESUMEN

- Artículo recibido en octubre 2019 - Arbitrado en noviembre 2019 - Publicado en enero 2020

Durante la facilitación del curso Lenguaje y Percepción Musical de la carrera Educación Musical del Instituto Pedagógico de Miranda de la UPEL, se detectaron deficiencias de aprendizaje a causa del desencuentro entre los conocimientos musicales autodidacta y académico de los estudiantes. Con el propósito de integrar dichos conocimientos se diseñó y aplicó una propuesta instruccional en tres sesiones estratégicas para el desarrollo rítmico, melódico y armónico. La modalidad empleada fue el proyecto factible, apoyado en una investigación de campo con una metodología mixta de carácter explicativo secuencial. Se utilizó como técnica de recolección de datos la encuesta y como instrumento el cuestionario. Luego del análisis de los resultados del diagnóstico, la elaboración, presentación del diseño instruccional original y la experiencia de aula, se experimentó el proceso de aprendizaje de los elementos de la música a partir de la relación entre sus conocimientos previos con la nueva información académica.

Palabras clave: Conocimiento musical autodidacta; conocimiento musical académico; estrategias de enseñanza; Lenguaje y Percepción Musical

\section{ABSTRACT}

During the facilitation of the Language and Musical Perception course of the Musical Education career of the Instituto Pedagógico de Miranda of UPEL, learning deficiencies were detected due to the mismatch between the students' self-taught and academic musical knowledge. With the purpose of integrating this knowledge, an instructional proposal was designed and applied in three strategic sessions for rhythmic, melodic and harmonic development. The modality used was the feasible project, supported by a field investigation with a mixed methodology of sequential explanatory character. The survey was used as a data collection technique and the questionnaire as an instrument. After the analysis of the results of the diagnosis, the elaboration, presentation of the original instructional design and the classroom experience, the process of learning the elements of music was experienced from the relationship between their previous knowledge with the new academic information.

Keywords: Self-taught musical knowledge; academic musical knowledge; teaching strategies; Language and Musical Perception 


\section{INTRODUCCIÓN}

La praxis docente en todos sus niveles y sobre todo para quienes se forman en el ámbito pedagógico, representa un acto en el cual se conjugan la vocación, la creatividad, así como la constante búsqueda y atención en cuanto a los avances de la educación en lo social, político y cultural. En dicha búsqueda, el proceso de investigación representa un papel determinante del que dependerá la efectividad y el avance para dar respuesta a las necesidades y problemáticas en los procesos de enseñanza y de aprendizaje, en todas las áreas del conocimiento en la que se desarrolle el hecho educativo.

En este caso, se trabajó con la música como un arte de expresión de sentimientos y pensamientos en el entorno social y como factor importante en la formación del ser humano. Desde sus orígenes, la música se manifiesta como un acto intuitivo e imitativo humano por la necesidad de comunicación con el ambiente; el hombre imitaba los sonidos del entorno y se comunicaba a través de sonidos con diferentes tipos de modulaciones: graves y agudas, prolongadas y cortas, ásperas y suaves (Gentile, 2011).

Por estas razones, grandes pensadores han propuesto la música como parte del proceso formativo debido a que la música está ligada al orden, la armonía, la proporción y el equilibrio, por lo tanto es un complemento ideal que genera experiencias estéticas (Leiva y Mates, 2002, p. 276). Igualmente, los autores citados afirman que la música es un arte, una ciencia y una técnica que promueve en el ser humano los afectos, la receptividad y la atención, así como desarrolla procesos de abstracción, razonamiento lógico y matemático, la imaginación, el orden, la creatividad y el perfeccionamiento de los sentidos.

De acuerdo con lo anteriormente expuesto, la educación musical es considerada como un modo y un camino para el desarrollo integral del ser humano, razón por la cual se trabaja mundialmente en función de integrarla en el hecho educativo como un proceso globalizador que desarrolle la expresión, la comunicación, el entendimiento, la creatividad, la imaginación, la improvisación y el buen vivir (Rodríguez, 2003).

Con el mismo propósito de generar conciencia acerca del valor de la música en el desarrollo del ser y de la sociedad, en Venezuela se incluyó la educación musical como parte de los estudios de formación universitaria. Desde 1983, se crea la mención Música en la Universidad de Carabobo (UC) y en la Universidad Pedagógica Experimental Libertador (UPEL), donde en 1997 se revisó el "diseño curricular y se estableció que el título que otorga dicha institución a partir de ese año es el de Profesor de Educación Musical (...) equivalente a licenciado" (García, 2009, p. 16). 
Al facilitar cada curso en los diferentes periodos académicos de la especialidad, se ha encontrado que los contenidos que conforman el curso Lenguaje y Percepción Musical, ubicado en el primer periodo académico, son prerrequisitos para los demás cursos pertenecientes a la matriz de ubicación y secuencia de la especialidad. Es decir, este curso es el eje trasversal de la especialidad ya que se propone desarrollar en el estudiante: "los conocimientos y destrezas en el dominio teórico-práctico del lenguaje musical, enmarcado dentro de un contexto metodológico, apoyado en las tendencias pedagógicas musicales modernas que capacitarán al alumno para utilizarlas en los diversos niveles del sistema educativo" (Linares, 2005, p. 1).

Durante la administración del curso señalado, se ha detectado que los estudiantes presentan algunas deficiencias de aprendizaje cuya posible causa sea el desencuentro entre el conocimiento musical autodidacta y el nuevo conocimiento musical académico a adquirir.

Conviene destacar que durante el proceso investigativo surgieron las siguientes interrogantes: ¿Cuál es el nivel de conocimiento musical autodidacta y académico que poseen los estudiantes del curso Lenguaje Percepción Musical de la carrera de Educación Musical de la UPEL-IPM? ¿Cuáles estrategias de enseñanza se podrían aplicar para promover el vínculo entre los conocimientos musicales autodidacta y académico de los estudiantes para la compresión y el aprendizaje en este curso? ¿Qué efectos pudiera tener la aplicación de las estrategias de enseñanza en el aprendizaje musical de los estudiantes? y ipodría diseñarse un material instruccional destinado, entre otros fines, a integrar todos estos conocimientos?

A partir de esta serie de preguntas, la investigación se trazó como objetivo general diseñar estrategias de enseñanza que propicien el vínculo entre los conocimientos musicales autodidacta y académico para la compresión y aprendizaje de los contenidos de un curso de Lenguaje y Percepción Musical, en estudiantes de Educación Musical de la Universidad Pedagógica Experimental Libertador, del Instituto Pedagógico de Miranda José Manuel Siso Martínez (Linares, 2016).

Esta propuesta estratégica representa, en la especialidad de Educación Musical, un aporte didáctico y teórico importante, ya que muestra una visión creativa e innovadora de la enseñanza del lenguaje y la percepción musical, adaptada a las necesidades y procesos de aprendizaje de los estudiantes sujetos de investigación.

Debe destacarse que en el campo de la investigación sobre pedagogía musical existe originalidad e innovación con producción, diseño y propuestas de trascendencia para la interacción y alianza entre el conocimiento profesional 
musical y la pedagogía, por medio de actividades con modelo estratégico y creativo.

Los estudios comparativos que contribuyen al aprendizaje efectivo de los instrumentos musicales (Dávila, 2011) y los trabajos interdisciplinares que proveen herramientas centradas en actividades musicales pertinentes $\mathrm{y}$ creativas en el nivel inicial (Gallegos, 2010) aportan estrategias en consonancia con las nuevas corrientes pedagógicas. Uribe (2010), por ejemplo, detectó debilidades de comprensión musical en el aspecto histórico, comunicativo y social, especialmente, un desconocimiento de códigos estéticos, quinésicos y lingüísticos que forman parte de la obra musical y de sus componentes interpretativos esenciales. Se comprende, entonces, que la planificación y desarrollo pedagógico se haya planteado la enseñanza a partir del enfoque estratégico que promueve, simultáneamente, con el conocimiento y el aprendizaje, el proceso formativo en el ser humano durante su ciclo vital.

Durante la investigación fue propicio valorar el enfoque del aprendizaje sociocultural que, según Ángeles (2003), expresa que el alumno será capaz de lograr su aprendizaje por medio de la planificación de actividades, apoyado en otros aprendices con un nivel de conocimiento más avanzado; este proceso de intervención y tutelaje debe ser acompañado por los docentes, quienes al inicio crearán el ambiente con aportes estratégicos que generen en ambos aprendices el sentido del diálogo y trabajo grupal, para luego dejarlos a cargo del proceso hasta que se logre un manejo con más autonomía y autorregulación del aprendizaje.

Dependerá del docente y su organización de la instrucción, la creación de las zonas de desarrollo próximo (ZDP) por parte del estudiante (Vygotski, 1995), que según Ángeles (2003) tienen como propósito "relacionar lo que es capaz de hacer ahora con lo que será capaz de hacer mañana con el apoyo de otros individuos más capaces" (p. 31). Todo esto llevado a cabo a través de la organización de sistemas de andamiaje viables y didácticos.

Para Ausubel, Novak y Hanesian (1983), el aprendizaje del alumno dependerá de la estructura cognitiva previa que se relaciona con la nueva información, entendiendo como estructura cognitiva el conjunto de conceptos e ideas que un individuo posee en un determinado campo del conocimiento. Es decir, que el aprendizaje significativo se procura cuando los contenidos son relacionados con los que el alumno ya sabe; dicho proceso se llevará a cabo si el individuo presenta en su estructura cognitiva conceptos, ideas y proposiciones que podrán interactuar con la nueva información. Así, este aprendizaje (Hernández, 1998) produce una retención más duradera de la información y facilita la adquisición de 
nuevos conocimientos relacionados con los anteriormente adquiridos. De esta forma, según Ausubel, Novak $\mathrm{y}$ Hanesian (1983) el aprendizaje de conceptos y proposiciones se une con el aprendizaje subordinado, es decir, cuando existe una relación de subordinación entre el nuevo material y la estructura cognitiva, lo aprendido será duradero.

A partir de estas teorías educativas en el diseño y aplicación de las estrategias de enseñanza en el curso de Lenguaje y Percepción Musical I se pretendió: a) establecer los diferentes niveles de conocimiento musical y formación autodidacta y académica para diseñar las estrategias de enseñanza sobre la base de la música que conocen en el nivel de representación auditiva, visual $\mathrm{y}$ kinestésico y lograr la conexión de dichos conocimientos; b) promover el aprendizaje y práctica en equipo de los elementos de la música entre estudiantes con formación musical autodidacta $\mathrm{y}$ académica, así como también con diversidad de niveles de conocimientos musicales; c) en lo que atañe a la enseñanza del ritmo, relacionar cada esquema con palabras conocidas para lograr un aprendizaje rítmico progresivo, hacer ejercicios de polirritmia, ejercicios con voz y cuerpo de manera individual y grupal con ritmos afrovenezolanos $\mathrm{y}$ otros ritmos conocidos; d) para la enseñanza de la melodía, practicar ejercicios de escalas de manera individual y grupal y ejercicios de transcripción de melodías de canciones conocidas venezolanas y universales (valses, merengues, reguetón, pop latino) y e) para la enseñanza de la armonía, realizar ejercicios de escalas mayores y menores a dos y tres voces, organizados en uno, dos y tres grupos de estudiantes con diferentes niveles de conocimiento musical, además de ejercicios de improvisación armónica con melodías conocidas (Segundas voces)

Ahora bien, ¿de qué dependerá su éxito o fracaso? Pues de la organización, diseño, evaluación reflexiva, creatividad e innovación de las pautas, contenidos, recursos, momentos y actividades que los docentes tengan en cuenta al momento de ejecutarlo; y a favor de lo antes descrito, se comulga con las ideas de Alfonzo (2003), cuando plantea que:

El proceso de enseñanza, que conduce el docente debe captar y mantener la atención de los estudiantes, guiarlos a alcanzar objetivos de aprendizaje concretos, alentarlos durante el proceso $\mathrm{y}$, retroalimentarlos, entonces, tendremos una situación multivariada que requiere de una planificación sistemática y flexible que le dé respuestas pertinentes a este complejo proceso. (p. 2)

Para Díaz y Hernández (2010), las estrategias de enseñanza son el medio y el recurso que prestan ayuda pedagógica en función de las necesidades de los estudiantes para la mejora constructiva y el desarrollo de su aprendizaje. Anijovich y Mora 
(2009) las consideran como "el conjunto de decisiones que toma el docente para orientar la enseñanza con el fin de promover el aprendizaje de sus alumnos" (p. 4). En definitiva, se podría afirmar que estas estrategias son las diferentes tareas, actividades $\mathbf{O}$ acciones reflexivas organizadas $\mathrm{y}$ modeladas secuencialmente por el docente, con sentido creativo e innovador para el logro del aprendizaje efectivo, productivo, colaborativo $\mathrm{y}$ significativo en sus alumnos.

En cuanto al diseño de las distintas estrategias de enseñanza musical, se fundamentó en la metodología de enseñanza musical propuesta por los maestros y pedagogos Emile Dalcroze y Carl Orff.

Para Dalcroze (Instituto Emile Jaques Dalcroze, 2010; Sanjosé, 2003; Yépez, 2011) son fundamentales la observación, análisis y reflexiones en el hecho educativo y en el medio profesional artístico. Dalcroze propuso ejercicios rítmicos utilizando su propio cuerpo a manera de reforzamiento del conocimiento intelectual; precisó que el ritmo es el elemento esencial para la formación musical, ya que por medio de este se sientan las bases para la creación, ejecución melódica y armónica del lenguaje y la percepción de los sonidos. Sus planteamientos establecen que es obligación del docente el desarrollo rítmico, melódico y armónico durante la actividad musical en el aula y estar presente en el proceso de aprendizaje musical del alumno con actividades que despierten sus sentidos, todo su cuerpo como una orquesta y su impresión estética para una mejor compresión de la música.

El solfeo que propone Dalcroze desarrolla el oído interno para escuchar musicalmente y cantar afinadamente. Los conceptos musicales se refuerzan a través de la interacción física y auditiva. La práctica solfística va más allá de la idea de un aprendizaje gramatical lleno de reglas y prohibiciones; lo concibe como un despertar del sonido de los grados y las relaciones de altura entre los sonidos y la facultad de reconocer diversos timbres. A través del método, pretende enseñar a los estudiantes a oír y a representar mentalmente las melodías y combinaciones de toda naturaleza, a descifrarlas y a improvisar vocalmente, a anotarlas y componerlas. También, desarrolla el análisis del sentido armónico procurando la audición interior.

Con respecto a la metodología de Orff (Fundación "Carl Orff", 1984; Sanjosé, 2003; Yépez, 2011), su fundamento pedagógico se basa en el aprendizaje musical sobre los elementos que originan la combinación sonora en el aprendiz, de acuerdo con sus condiciones psicológicas (formas de ser y de comportarse). Sobre la base de lo antes planteado Orff propone la educación musical por medio del análisis y conocimiento de la naturaleza 
del sonido y el ritmo desde la percepción humana. El aprendiz desarrolla el lenguaje y la percepción musical a través de la base cultural aprendida en el entorno escolar y familiar (himnos, rondas, canciones infantiles y escolares aprendidas en el aula, entre otras). Considera palabra, música y movimiento como un solo componente aplicado de modo real y consciente para el desarrollo integral del ser (Sanjosé, 2003).

Para Orff (Yépez, 2011) el desarrollo rítmico toma como eje principal la célula generadora del ritmo en la palabra, inicia con el recitado de nombres, frases, palabras del acervo cultural. Se parte de la palabra para llegar a la frase, esta frase es transmitida al cuerpo transformándolo en un instrumento para trabajar la denominada "percusión corporal" para pasar progresivamente a la percusión instrumental. Promueve el desarrollo de la improvisación rítmica por medio de ejercicios de ecos y palmadas, combinaciones entre ambos, pisadas, pitos, palmas en rodillas y luego se trabajará con el uso de los diversos instrumentos de percusión llamados orquesta Orff. Finalmente, el aprendizaje rítmico por medio de las negras, por ser el pulso más natural, y por ampliación o reducción surgen las otras figuras de notas. La melodía se estudia con canciones populares infantiles: a) recitados rítmicos verbales (poesía, adivinanzas), que se acompañan con instrumentos naturales; b) creación de una melodía para el recitado rítmico o rítmico verbal; c) se añaden sencillos patrones melódicos con la voz y con los instrumentos; d) improvisación por medio de ecos melódicos, donde dos o tres notas sugeridas realizan la improvisación.

Se utilizan el canon y las composiciones polifónicas como recurso para el desarrollo de la imitación melódica; composición melódica sobre la base de dos, tres, cuatro o cinco sonidos creados por el aprendiz en niveles superiores; creaciones melódicas para un texto y la creación de un texto para una melodía.

Para la armonía se utilizan ejercicios de improvisación armónica sobre el esquema del canto homofónico, organum y discantus; ejercicios armónicos con los instrumentos de percusión afinada tales como xilófonos, metalófonos, carrillones, y la viola da gamba; acompañamiento en las canciones y danzas; creación armónica sobre la base de acompañamiento con ostinatos por los grados Tónica (I) y Dominante (V); creación de melodías paralelas y contramelodías sobre una melodía o cantus firmus ya realizado.

Estos métodos de enseñanza musical de Dalcroze y Orff, a efectos del enfoque estratégico de las actividades de enseñanza, son los más acertados para el logro del objetivo planteado del presente trabajo. Estas propuestas se conjugaron con el fin máximo referido a la conexión de los conocimientos 
musicales autodidacta y académico por medio del diseño y la aplicación de las estrategias de enseñanza musical para el desarrollo rítmico, melódico, y armónico en los estudiantes de la UPEL-IPM.

Por otra parte, en el marco de la problematización argumentada del proceso investigativo, se estimó que el conocimiento musical autodidacta y académico así como su correspondencia, representan el proceso que hace posible el aprendizaje del lenguaje y la percepción musical por medio del diseño de las estrategias de aprendizaje.

Para Keller (2014) el conocimiento autodidacta "está muy ligado a la música popular, casi siempre son conocimientos o destrezas que se transmiten de persona a persona. Los resultados, generalmente, dependen de su propio empeño"(s/n). Mientras que el conocimiento académico "es aquel que se recibe en una escuela de Música, sujeto a un programa generalmente elaborado para preparar músicos profesionales. Es la base de todo músico profesional que quiera dedicarse a la llamada "música clásica", pero también puede ser la formación de cualquier otra rama de la música". (Keller, entrevista personal, 2014)

El conocimiento musical autodidacta es el que se genera del aprendizaje propio del canto, un instrumento particular, el aprendizaje y creación de melodías; todo esto desde los tres sistemas de representación: auditivo (de guataca o de oído, términos populares que se atribuye a este tipo de aprendizaje musical), visual (viendo, termino referido al aprendizaje musical por medio de la observación al experto ya sea en el área vocal o instrumental), kinestésico (dándole o charrasqueando, términos populares que se refieren al proceso de aprendizaje del instrumento por la práctica por la observación al experto). Mientras que el conocimiento musical académico produce comprensión y aprendizaje por medio de la instrucción docente calificada (conservatorios, academias, talleres de formación itinerantes, universidades).

\section{MÉTODO}

El tipo de investigación estuvo determinado bajo la modalidad de Proyecto Factible llevado al campo. Este proyecto "consiste en la investigación, elaboración y desarrollo de una propuesta de un modelo operativo viable para solucionar problemas, requerimientos $\mathrm{o}$ necesidades de organizaciones o grupos sociales; puede referirse a la formulación de políticas, programas, tecnologías, métodos o procesos" (UPEL, 2016, p. 21).

De acuerdo con Arias (2006), la investigación de campo es un proceso que "consiste en la recolección de datos directamente de los sujetos investigados, o de la realidad donde ocurren los hechos (datos primarios) sin manipular o controlar variable alguna" (p. 31). 
Se realizó una fase diagnóstica durante la facilitación del curso Lenguaje y Percepción Musical, en la que se precisaron distintas deficiencias que interfieren en el proceso de aprendizaje de los estudiantes sujetos de investigación, desde lo conceptual (falta de actividades didácticas en los materiales de estudio); desde lo procedimental (falta de conocimiento los estudiantes sobre el recurso que representa la música popular para el aprendizaje al igual que la música académica), y desde lo actitudinal (falta de valoración y reconocimiento por la música académica y popular como fuente de conocimiento). En la fase de factibilidad se analizaron las causas que generan estas dificultades y la viabilidad de realizar aplicaciones que subsanen las falencias en el propio ambiente. La fase de diseño de la propuesta se procedió con el diseño de instrucción, en el cual se desarrollan estrategias de enseñanza para lograr la conexión entre los conocimientos autodidacta y académico.

En el proceso de investigación se aplicó el diseño mixto de carácter explicativo secuencial que, de acuerdo con lo planteado por Hernández, Fernández y Baptista (2010),

(...) se caracteriza por una primera etapa en la cual se recaban y analizan datos cuantitativos, seguida de otra donde se recogen y evalúan datos cualitativos. La mezcla ocurre cuando los datos cuantitativos iniciales informan sobre los resultados de los datos cualitativos. Finalmente, los descubrimientos de ambas etapas se integran en la interpretación y elaboración del reporte del estudio. (p. 566)

La fase diagnóstica se efectuó de modo cuantitativo para determinar el nivel de conocimiento musical declarativo, procedimental y actitudinal de los estudiantes. Sobre la base de los resultados arrojados en el diagnóstico, se procedió al diseño de las estrategias de enseñanza musical. Luego de la aplicación de las estrategias, en la fase cualitativa se analizaron los resultados e impresiones para luego integrar ambas fases y llegar a las conclusiones del proceso de enseñanza estratégica.

Los sujetos de estudio fueron los estudiantes de un grupo del curso Lenguaje y Percepción Musical I del IPM - UPEL. Estuvo conformado por catorce (14) participantes de edades comprendidas entre dieciocho (18) y cincuenta (50) años, procedentes de liceos públicos y privados, conservatorios y academias de música, ministerios de música religiosa y orquestas pertenecientes al Sistema Nacional de Orquestas y Coros Juveniles e Infantiles de Venezuela, docentes de música en ejercicio, músicos profesionales en ejecución vocal, instrumental y dirección coral. Todos sin formación pedagógica.

La estrategia de organización de contenido comprende tres momentos 
denominados instruccionales (inicio, desarrollo y cierre) y en cada momento se desarrollan a su vez varios eventos instruccionales. Según Smith y Ragan (como se citó en Alfonzo, 2003), deben propiciarse los siguientes procesos mentales: centrar la atención y percibir selectivamente la nueva información; almacenar la nueva información en la memoria a corto plazo; establecer relaciones entre el nuevo conocimiento y los que ya poseía el individuo; almacenar el nuevo conocimiento en la memoria a largo plazo.

La variable fundamental, por consiguiente, de esta investigación fue el conocimiento musical visto en tres dimensiones: declarativa, procedimental y actitudinal. Luego de recolectar los datos que sirvieron de base para diagnosticar el nivel de conocimiento de los participantes se procedió a confeccionar el programa con el conjunto de estrategias para llegar a fusionar el conocimiento autodidacta con el conocimiento académico.

Las técnicas e instrumentos para la recolección de datos se aplicaron por fases: inicial (cuantitativa): la técnica seleccionada fue la encuesta a través de un cuestionario como instrumento de recolección que de acuerdo con Hernández, Fernández y Baptista (2010), consiste en "un grupo de preguntas respecto a una o más variables a medir." (p. 217), este se utilizó para determinar el nivel de conocimiento musical de los estudiantes; la segunda (cualitativa) correspondió a la aplicación de la observación que consiste en "captar mediante la vista, en forma sistemática, cualquier hecho, fenómeno o situación que se produzca en la naturaleza o en la sociedad, en función de sus objetivos de investigación preestablecido" (Arias, 2006, p. 69); se utilizó un guion de observación como instrumento de recolección de la información.

La validez de los instrumentos se determinó por medio del juicio de tres expertos, uno en el área de Educación Musical con experticia en el enfoque estratégico, uno en el área de las Estrategias de enseñanza y uno en el de la evaluación educativa.

Adicionalmente, en el caso del cuestionario, se aplicó una prueba piloto para determinar la compresión de los ítems por parte una población similar a los sujetos de investigación.

\section{RESULTADOS}

El cuestionario con veinticinco ítems, redactados en función de las dimensiones (declarativa, procedimental y actitudinal), arrojó las siguientes derivaciones: en la dimensión declarativa se mostró que los estudiantes conocen 24 canciones y obras populares venezolanas, suramericanas, centroamericanas, norteamericanas y europeas, sumadas a 22 obras académicas de los mismos ámbitos, en especial obras venezolanas. Igualmente, conocen diversidad de estilos: folklórico, 
balada, gaita, joropo, merengue, oriental, rock, pop, salsa, y vals. Los instrumentos conocidos por sus respectivas familias fueron: aerófonos, cordófonos, idiófonos y membranófonos.

En la dimensión procedimental las canciones cantadas mencionadas en orden, por género fueron: académicas, latinas, populares, pop, rock, sacro, trova. Se encontró que las canciones populares ocuparon la mayor frecuencia. Se aprecia que $42 \%$ de los estudiantes interpretan canciones populares y de corte religioso, mientras que $25 \%$ interpreta canciones de corte académico y latino. En la misma dimensión, 78\% ejecuta instrumentos cordófonos.

En la dimensión actitudinal, el gusto por el género quedó en este orden: latino, popular, pop, rock, sacro, trova y jazz. Obviamente, informaron que su gusto se deriva del mayor conocimiento sobre ese género. Asimismo, en esta dimensión prefirieron las agrupaciones académicas nacionales que internacionales, por su conocimiento sobre ellas. Manifestaron un mayor gusto por los instrumentos cordófonos debido a su conocimiento y a causa de que tienen un valor estético, profesional $y$ en algunos casos sentimental en su carrera como docentes y artífices de la música.

Luego del análisis de los resultado de la aplicación del cuestionario y precisar la viabilidad de realizar aplicaciones que subsanen las falencias, se llevó a cabo la fase de diseño instruccional, en el cual se incluyen tres grandes estrategias para la enseñanza del ritmo, melodía y armonía con el fin de que los estudiantes logren relacionar sus conocimientos, práctica y valoración musical autodidacta, con el nuevo conocimiento musical académico para la compresión del curso Lenguaje y Percepción Musical.

Es importante señalar que la variedad de estilos de la música vocal e instrumental nacional e internacional, así como también cada instrumento musical, fueron clasificados de acuerdo con sus respectivos géneros y familias, $\mathrm{y}$ es de allí de donde se seleccionaron los más acordes para el diseño de las estrategias. Estos géneros musicales nacionales e internaciones, los instrumentos musicales y sus familias para proceder con el diseño de las estrategias de enseñanza musical, seleccionados a partir de los resultados, fueron:

En la estrategia de enseñanza para el desarrollo y aprendizaje del ritmo se trabaja, en un primer momento: a) el conocimiento teórico de las figuras de notas; b) para la música nacional: El becerrito, Simón Díaz, Género: Merengue venezolano. Parranda de San Pedro, Tradición oral, Género: Parranda. Sangueo del estado Carabobo, Género: Afrovenezolano. Tambor de San Millán, estado Carabobo, Género: Afrovenezolano; c) para la música internacional: I wanna hold your hand, The Beatles, Género: Rock. Tiempo de vals, Chayanne, Género: Pop. No es 
serio este cementerio, Mecano, Género: Pop. Te vi venir, Sin bandera, Género: Balada; d) instrumentos musicales: Teclado, Familia: Cordófonos. Tambora y paila, Familia: Membranófonos. Maracas y Laures, Idiófonos.

En la estrategia de enseñanza para el desarrollo y aprendizaje de la melodía: a) música nacional: Canto de sirena, estado Miranda, Género: Afrovenezolano. Calipso del estado Bolívar, Género: Calipso. E1 becerrito, Simón Díaz, Género. Merengue Venezolano. Moliendo café, Género: Orquídea; b) música internacional: Felina, Género: Reguetón; c) instrumentos musicales: Teclado, guitarra y cuatro. Familia: Cordófonos. Trombón y flauta: Familia: Aerófonos.

En la estrategia de enseñanza para el desarrollo y aprendizaje de la armonía: a) música nacional: Canto de lavanderas, estado Yaracuy, Género: Cantos de trabajo. Sangueo del estado Carabobo, Género: Afrovenezolano; b) música internacional: $\mathrm{Da}$ Pacem Domine, Género: Motete; c) instrumentos musicales: Teclado, guitarra, cuatro y viola. Familia: Cordófonos. Trombón y flauta. Familia: Aerófonos.

\section{Identificación, propósito y justificación de la propuesta}

El diseño instruccional estuvo conformado por tres estrategias para la enseñanza musical del ritmo, la melodía y la armonía. Tiene como finalidad ofrecer a los estudiantes una serie de actividades que generen, durante su proceso de aprendizaje, el vínculo entre conocimientos musicales producto de su experiencia autodidacta, con el nuevo conocimiento académico musical. Los participantes tienen diversos niveles de formación en el área de la música sin el componente pedagógico. Los docentes serán garantes del proceso del vínculo entre los conocimientos musicales autodidacta y académico para la compresión del curso Lenguaje y Percepción Musical I, propósito central de la propuesta. Y, del mismo modo, debe proponerse que los estudiantes con menor conocimiento musical logren el aprendizaje de los elementos de la música, por medio del estudio colaborativo con los estudiantes de mayor formación musical como sus referentes; igualmente, deben lograr en los estudiantes con mayor nivel de conocimiento musical, una aproximación al proceso de enseñanza musical desde la pedagogía y los enfoques $\mathrm{y}$ teorías educativas al momento del estudio y el trabajo en colaboración con los estudiantes de menor formación musical.

A continuación se presenta el resumen del diseño instruccional original.

\section{DISEÑO}

INSTRUCCIONAL

(Cuadros 1, 2 y 3)

\section{- Autor: Sergio Linares}

- Información académica 
- Curso: Lenguaje y Percepción Musical I.

- Objetivo general: desarrollar de manera progresiva el ritmo, la melodía y la armonía por medio de la conexión de los conocimientos autodidacta y académico, en los estudiantes del curso Lenguaje y Percepción Musical I.

\section{- Contenidos a facilitar en el curso:}

Rítmicos: figuras de notas compuestas: cuartina, galopa, contra galopa, saltillo y síncopa; Melódicos: las notas musicales; Armónicos: tonalidad mayor.
- Enfoque Psicológico: Cognitivo. Teorías de aprendizaje: Significativo (Ausubel). Sociocultural (Vygotski).

- Conocimientos previos: las figuras de notas (redonda, blanca, negra, y semi corchea) y sus silencios. Concepto de música. Elementos de la música y calidades del sonido. Estudios en institutos de música sin terminar. Estudios de en ejecución instrumental y dirección coral (estudiantes avanzados).

Cuadro 1. Estrategia para desarrollo rítmico

Estrategias de enseñanza para el aprendizaje y desarrollo rítmico

\section{PLAN DE CLASES}

Nombre de la Estrategia:

Tiempo:

Signos principales de la música (las figuras de notas)

180 minutos

Meta de aprendizaje:

Promover el desarrollo del oído rítmico y el conocimiento de las figuras de notas por medio de actividades lúdicas.

Contenidos:

Declarativos: Conocimiento teórico de las figuras de notas, duración, forma, y ubicación en el pentagrama.

Procedimentales: Realiza de manera individual y grupal diversos ejercicios por medio de juegos, con esquemas rítmicos del folklore venezolano ya conocidos. Actitudinales: Es consciente del ritmo, su utilidad en la educación musical y en la vida.

Estrategias de evaluación:

Actividad: Observación participante. Técnicas: La pregunta.

Instrumentos: Escala de estimación.
Sustentación teórica:

Alfonzo (2003)

Feo (2010)

Sanjosé (2003)

Yépez (2011)

Observaciones

Secuencia didáctica 


\begin{tabular}{lll}
\hline \multicolumn{1}{c}{ Momento } & \multicolumn{1}{c}{ Actividades } & \multicolumn{1}{c}{ Recursos } \\
\hline \multirow{3}{*}{ INICIO } & Activar la atención: Luego de saludar se pide escuchar un & Equipo de audio \\
& audio y ver videos de diferentes ritmos. Preguntar cuáles & Cd y video con \\
& conocen, cuándo los escucharon, qué recuerdo les trae y si es & $\begin{array}{l}\text { ritmos del } \\
\text { mundo. }\end{array}$ \\
& parte de su vida y por qué. & Ordenador \\
& Visión preliminar de la lección: exponer los contenidos a & portátil \\
Tiempo & desarrollar, las figuras de notas compuestas, sus formas, & Presentación \\
$\mathbf{3 0 ~ m i n u t o s}$ & duración y reproducción sonora. & Power Point \\
& Establecer el propósito: leer, reproducir de manera corporal y & Video Beam \\
& reconocer auditivamente las figuras de notas compuestas. & Cuaderno \\
& Incrementar el interés: presentar PPT de notas compuestas & Pentagramado \\
& (Cuartina, saltillo, galopa y contragalopa) y preguntar si son & Lápices de grafito \\
& conocidas, si consideran difícil su aprendizaje y por qué. Luego & Borrador
\end{tabular}
antes expuesto.

Recordar conocimientos previos: preguntar sobre notas conocidas, pasar al pizarrón estudiantes para dibujar y

DESARROLLO reproducir notas y silencios corporalmente; pedir a otro estudiante que recuerde el valor de cada figura de nota con la fórmula dada.

Procesar la nueva información: Ver las notas en PPT.

Tiempo 40 minutos

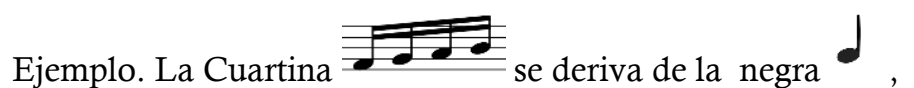
modela con voz y palmas lo antes expuesto y pide a la audiencia que repita el ejemplo modelado.

Estrategias de aprendizaje y práctica: escribir cada figura de nota vista en PPT en sus cuadernos pentagramados; los estudiantes avanzados supervisan y apoyan. Se pide a cada alumno realizar un ejercicio de 10 compases rítmicos con las figuras de notas vistas y que luego las reproduzca con una parte del cuerpo (Dalcroze).

Focalizar la atención: se organizan dos grupos para ejecutar un ejercicio con las figuras de notas vistas. El primer grupo lee un compás y el segundo grupo lee el siguiente y así sucesivamente. Luego, se repite el ejercicio con tres grupos y finalmente, de uno en uno (Orff). Al terminar esta dinámica el docente da 10 minutos de receso.

Transferencia de los aprendizajes: preguntar cómo suena cada figura de nota, con palmas y voz; el docente dice las figuras con palmas y voz para que discriminen y escriban en su cuaderno pentagramado qué figura de nota está reproduciendo. Colocar un audio con canciones de varios géneros conocidos y pedir que señalen en qué momento de la canción aparecen las figuras de notas aprendidas y que las reproduzcan. Por último pedir a un estudiante (percusionista) que ayude a cada uno, sobre un ritmo afrovenezolano, a improvisar figuras de notas vistas antes y durante la clase.

Revisar y resumir la lección: preguntar cómo se sintieron, si hubo dificultades y cómo las resolvieron, si hubo apoyo de cada compañero y de qué forma.

Proponer enlaces: pedir que lean el escrito que hicieron al comienzo y que contrasten si realmente les resultó difícil o divertido el proceso de aprendizaje y qué herramientas les da la clase para su praxis docente.
Equipo de audio

Cd con canciones de distintos géneros nacionales e internacionales Instrumentos de percusión afrovenezolana

Cuaderno pentagramado. Lápices de grafito Borrador 


\section{Cuadro 2. Estrategia para desarrollo melódico}

Estrategia de enseñanza para el aprendizaje y desarrollo melódico

\section{PLAN DE CLASES}

Nombre de la Estrategia:

Tiempo:

Las notas musicales, los movimientos e intervalos musicales.

$180 \mathrm{~min}$

\section{Meta de aprendizaje:}

Promover el desarrollo del oído melódico y el conocimiento de las notas musicales, movimientos e intervalos musicales en tonalidad mayor por medio de actividades lúdicas

Contenidos:

Declarativos: Conoce las notas musicales, nombre, posición en la ubicación en el pentagrama.

Procedimentales: Realiza, de manera individual y grupal, diversos ejercicios por medio de la entonación y lectura de canciones del folklore venezolano y géneros internacionales.

Actitudinales: Toma conciencia y fomenta el valor de la melodía, su utilidad en la educación musical y en la vida.

Estrategias de evaluación:

Actividad: Observación participante.

Técnicas: La pregunta. Instrumento: Escala de estimación.

\section{Secuencia didáctica}

\section{Momento}

INICIO

\section{Actividades}

Activar la atención: el docente saluda a la audiencia y de repente entran al salón dos alumnos de períodos avanzados: el primero canta varias melodías de diferentes géneros, y seguidamente entra otro con un instrumento haciendo lo mismo. Terminan y se retiran sin decir palabra.

Incrementar el interés: El docente pregunta a la audiencia ¿Cómo se sintieron con lo ocurrido? ¿Cuáles recuerdos trajo las melodías escuchadas? ¿Qué elementos sonoros estuvieron presentes?

Establecer el propósito: El docente, luego de escuchar a la audiencia, expone que al finalizar serán capaces de leer y entonar de manera progresiva las notas, la escala musical y los diferentes intervalos melódicos.

Visión preliminar de la clase: Al establecer el propósito el docente enuncia el contenido de la clase
Alfonzo (2003)

Feo (2010)

Sanjosé (2003)

Yépez (2011)
Sustentación teórica:

\section{Observaciones:}

\section{Recursos}

Equipo de audio.

Dos estudiantes de la especialidad en periodos avanzados.

Pizarrón pentagramado.

Marcadores para acrílico.

Cuaderno pentagramado. Lápices de grafito. Borrador. 


\begin{tabular}{|c|c|}
\hline Momento & Actividades \\
\hline DESARROLLO & $\begin{array}{l}\text { Recordar conocimientos previos relevantes: Se escribe en } \\
\text { el pizarrón un cuadro S.Q.A y pregunta si saben de qué se } \\
\text { trata. Luego explica el propósito del cuadro y solicita que lo } \\
\text { cumplimenten y lean. }\end{array}$ \\
\hline & $\begin{array}{l}\text { Procesa la nueva información y sus ejemplos: se pide una } \\
\text { reunión en grupos de tres (un avanzado con dos en } \\
\text { proceso); buscar en libros de teoría musical y comentar } \\
\text { grupalmente qué son la melodía, las notas musicales los } \\
\text { movimientos e intervalos melódicos. El dicente supervisa la } \\
\text { actividad. }\end{array}$ \\
\hline
\end{tabular}

Focalizar la atención: Al terminar, el docente pide a cada equipo que manifieste lo investigado. Luego se sienta al piano y con base en los resultados obtenidos, toca y a su vez enuncia las notas musicales para que los estudiantes las discriminen de manera auditiva. Luego, da un receso de 10 $\min$.

Estrategias de aprendizaje y práctica: se organizan y con un manual de lectura musical se lleva a cabo la estrategia calibre interválico, que consiste en entonar las notas de la escala de do mayor, y luego por intervalos de $2 \mathrm{da}$, 3ra, 4ta, $5 \mathrm{ta}, 6 \mathrm{ta}, 7 \mathrm{ma}$ y $8 \mathrm{va}$; aclarando que si hay dudas con respecto a la afinación de intervalo deben resolverlas con notas de paso. Esta estrategia permite activar la audición interior y la afinación en el discurso melódico (Dalcroze).

CIERRE Revisar y resumir la lección: Al terminar se pregunta cómo se sintieron con la práctica melódica, si hubo dificultades y Tiempo 30 minutos de qué forma.

Transferencia de los aprendizajes: El docente escribe en el pizarrón de acuerdo a los gustos de la audiencia, el nombre de varias canciones, pide que escojan una y solicita reunión en equipo de un estudiante avanzado y uno principiante para transcribir la melodía de 10 o 15 primeros compases de una canción, solo en notas musicales, con ayuda de un instrumento musical. Como segunda estrategia se promueve la entonación de escalas e intervalos en forma de eco. Se reúnen inicialmente dos, luego tres y finalmente cuatro. Un grupo comienza entonando la primera nota de la escala y el otro entona la segunda y así sucesivamente, cambiando de orden al momento de cada entonación. Esta estrategia genera concentración y trabajo en equipo (Orff).

Remotivar y cerrar: El docente pide a la audiencia que saque su cuadro S.Q.A para contrastar y reflexionar sobre el aprendizaje obtenido.

Proponer enlaces: Después de la discusión del cuadro S.Q.A. se pregunta sobre los aportes de las prácticas y estrategias. Se indica para la siguiente clase revisar sobre la armonía.

Cuadro S.Q.A.

Pizarrón pentagramado.

Instrumentos

musicales.

Cuaderno pentagramado.

Lápices de grafito.

Borrador.

Fuente: Elaboración propia. 


\section{Cuadro 3. Estrategia para desarrollo melódico}

Estrategia de enseñanza para el aprendizaje y desarrollo armónico

\section{PLAN DE CLASE}

Nombre de la Estrategia: Los grados de las escalas diatónica y cromática y la

Tiempo: armonía.

$180 \mathrm{~min}$

Meta de aprendizaje:

Promover el desarrollo del oído melódico y armónico a través de la entonación de la escala diatónica, la escala cromática y la lectura a dos, tres y cuatro voces.

\section{Contenidos:}

Declarativos: Conoce los grados de la escala diatónica, su posición y su ubicación en el pentagrama. Conoce la escala cromática.

Procedimentales: Realiza de manera individual y grupal diversos ejercicios de lectura, en dúos, tríos y cuartetos, de composiciones Venezolanas e Internacionales. Armoniza canciones y fragmentos melódicos.

Actitudinales: Toma conciencia y fomenta el valor de la armonía, su utilidad en la educación musical y en la vida.

Estrategias de evaluación:

Actividad: Observación participante

Técnicas: La pregunta

Instrumentos: Escala de estimación

\section{Secuencia didáctica}

Momento

Actividades

Activar la atención: Al entrar al salón estarán 4 estudiantes de

INICIO sexo femenino de cursos avanzados ubicadas en cada esquina. El docente dará la bienvenida y preguntará sobre la clase pasada; de repente las estudiantes desde sus esquinas

Tiempo 30 minutos interpretarán un canto de lavanderas y cuando estén por terminar irán saliendo del salón de clases, al finalizar dirán "Viva la música".

Incrementar el interés: al terminar se pregunta ¿Qué impresiones tienen de lo sucedido? ¿Habían escuchado este canto alguna vez? ¿Qué creen que vamos a ver en la clase de hoy? Y otras preguntas de interés y motivación.

Establecer el propósito: se enunciará el tema de la clase y al final se reconocerá qué es una escala diatónica, el nombre de sus grados, la entonación de melodías a varias voces y la creación de voces alternas a esas melodías.

Visión preliminar del contenido: se enuncia el contenido al establecer el propósito

\section{Sustentación} teórica:

Alfonzo (2003)

Feo (2010)

Sanjosé (2003)

Yépez (2011)

\section{Observaciones:}

\section{Recursos}

4 alumnas de nivel avanzado de la carrera Educación musical

Pizarrón pentagramado Marcadores para acrílico 


\begin{tabular}{|c|c|c|}
\hline Momento & Actividades & Recursos \\
\hline \multirow[t]{4}{*}{$\begin{array}{c}\text { Tiempo } \\
\mathbf{4 0} \text { minutos }\end{array}$} & $\begin{array}{l}\text { Recordar conocimientos previos relevantes: Preguntar } \\
\text { ¿Qué se vio en la clase pasada? ¿Qué relación tiene el tema } \\
\text { anterior con el nuevo tema de la clase? } \\
\text { Procesar la nueva información y sus ejemplos: tres } \\
\text { estudiantes avanzados pasan a la pizarra y exponen los } \\
\text { nombres de los grados de la escala diatónica, el porqué de } \\
\text { sus nombres y la escala cromática con sus respectivos } \\
\text { ejemplos. Luego, el docente pregunta y aclara dudas, } \\
\text { reforzará la intervención de cada expositor y recordará la } \\
\text { sencillez y didáctica del lenguaje al explicar este tipo de } \\
\text { contenidos. }\end{array}$ & $\begin{array}{l}\text { Pizarrón } \\
\text { pentagramado } \\
\text { Marcadores para } \\
\text { acrílico } \\
\text { Partitura polifónica } \\
\text { del siglo XVI } \\
\text { Cuaderno } \\
\text { pentagramado } \\
\text { Lápiz de grafito }\end{array}$ \\
\hline & $\begin{array}{l}\text { Actividades de aprendizaje y práctica: realizar ejercicios } \\
\text { de entonación con las diferentes escalas mayores haciendo } \\
\text { transporte melódico; se pide que construyan las diferentes } \\
\text { escalas diatónicas en modo mayor, la escala cromática de } \\
\text { re y fa mayor, y a los estudiantes avanzados que } \\
\text { monitoreen. Luego, se pregunta ¿Cómo fue el proceso de } \\
\text { elaboración de las escalas? ¿Si hubo dificultad se resolvió? } \\
\text { Preguntar los signos principales y secundarios utilizados } \\
\text { para la elaboración de las escalas. }\end{array}$ & \\
\hline & $\begin{array}{l}\text { Procesa la nueva información y sus ejemplos: el docente } \\
\text { escribe la palabra "armonía" en la pizarra y pide que la } \\
\text { definan de acuerdo con su experiencia musical con } \\
\text { ejemplos; preguntar la relación con el ritmo y la melodía. } \\
\text { Práctica melódico-armónica: Se pide que realicen algunas } \\
\text { escalas diatónicas y cromáticas a manera de canon, en } \\
\text { pares y tríos, cambiando el orden cada vez que se cante la } \\
\text { escala para trabajar la afinación y la concertación. }\end{array}$ & \\
\hline & $\begin{array}{l}\text { Focalizar la atención y proponer enlaces: se entrega a los } \\
\text { alumnos una partitura polifónica para grupo mixto del } \\
\text { siglo XVI y se organiza a los estudiantes en cuatro grupos, } \\
\text { se pide que identifiquen en qué tonalidad está la pieza y } \\
\text { cómo la identificaron. Después se pide que lean las notas } \\
\text { musicales, de manera rezada, presentes en cada voz. } \\
\text { Pregunta si hay parecido melódico de las demás voces y } \\
\text { cuáles voces tienen la mismas notas musicales en su } \\
\text { melodía. Luego solicita que lean el ritmo unas dos veces y } \\
\text { finalmente cada voz con ritmo, melodía y armonía. Esta } \\
\text { estrategia trabaja la desfragmentación de los elementos de } \\
\text { la música y la audición externa para una mejor } \\
\text { compresión y aprendizaje del lenguaje y la percepción } \\
\text { musical. Después de esta actividad se dan } 10 \text { minutos de } \\
\text { receso. }\end{array}$ & \\
\hline
\end{tabular}




\begin{tabular}{|c|c|c|}
\hline Momento & Actividades & Recursos \\
\hline $\begin{array}{c}\text { Tiempo } \\
30 \text { minutos }\end{array}$ & $\begin{array}{l}\text { Transferencia de los aprendizajes: El docente se sienta al } \\
\text { piano, pide a cada alumno que, con un acompañamiento } \\
\text { hecho a partir de un género popular e internacional } \\
\text { conocido, improvise una melodía. Como segunda } \\
\text { actividad indica que cada alumno haga una nota pedal y } \\
\text { que otro cree un segunda voz. Como estrategia final, pide } \\
\text { que canten una canción de su gusto acompañados de } \\
\text { instrumentos de cuerda (cuatro, guitarra, en diferentes } \\
\text { tonalidades para trabajar cambios armónicos. (Orff) } \\
\text { Revisar y resumir la lección: Al terminar la aplicación de } \\
\text { las diferentes estrategias para trabajar melodía y armonía, } \\
\text { el docente pregunta a la audiencia ¿Cómo se sintieron con } \\
\text { la sesión de clases? ¿Si hay dudas o inquietudes sobre los } \\
\text { temas propuestos? ¿Si se logró el propósito de la clase y de } \\
\text { qué forma se logró? } \\
\text { Proponer enlaces: El docente manifiesta que esta sesión es } \\
\text { la última para este curso y recomienda a los estudiantes } \\
\text { buscar información sobre las escalas menores y } \\
\text { construcción de intervalos. De igual manera, manifiesta su } \\
\text { agrado por el desarrollo del curso y felicita a la audiencia } \\
\text { por su implicación y aprendizaje obtenido, y abre el } \\
\text { espacio para que la audiencia exprese sus impresiones } \\
\text { sobre el desarrollo del curso y el desempeño docente. }\end{array}$ & $\begin{array}{l}\text { Piano electrónico } \\
\text { Canciones de } \\
\text { diferentes géneros } \\
\text { Instrumentos de } \\
\text { cuerdas }\end{array}$ \\
\hline
\end{tabular}

Fuente: Elaboración propia.

La aplicación de las estrategias de enseñanza musical para el aprendizaje del ritmo, la melodía y la armonía a través del vínculo entre los conocimientos musicales autodidacta y académico se llevaron a cabo durante seis sesiones de clases, divididas en una sesión para la aplicación de la secuencia didáctica y otra sesión práctica para evaluar los aprendizajes obtenidos a raíz de cada aplicación (se evaluaron los aprendizajes en el tiempo reservado de los 180 minutos pautados; además puede ser variable de acuerdo con las necesidades del grupo). El éxito dependió en su mayor parte de las dinámicas, la disposición de los estudiantes, los cambios e innovaciones y del espíritu de cuerpo logrado en cada sesión. Debe señalarse que cada secuencia didáctica estuvo bajo la técnica de observación, por tres docentes especialistas en el área de la enseñanza estratégica y la Educación musical quienes hicieron reportes adicionales sobre la experiencia.

\section{CONCLUSIONES}

En la investigación se llegó a profundizar en lo referido a la parte musical autodidacta y académica que trae cada estudiante, indagar en sus conocimientos previos y poder apreciar toda la diversidad de insumos e ideas que dieron al investigador para el diseño de la enseñanza estratégica. 
A pesar de que existían diferentes niveles de formación musical, había entre los estudiantes una conexión en cuanto a los gustos y conocimiento de los géneros musicales. Esto demostró que la música puede lograr unificar criterios, sentimientos y emociones a pesar de las diferencias de edad y nivel de formación musical e intelectual.

Debe asumirse que durante la planificación diaria y constante sobre la base de estos enfoques y teorías se logró en los estudiantes un mejor desarrollo de la cognición y construcción del conocimiento y en el docente la compresión, la experticia y conciencia pedagógica.

Se pudo confeccionar, validar y aplicar el diseño de estrategias de enseñanza que propiciaron el vínculo de los conocimientos autodidacta $\mathrm{y}$ académico para la compresión $\mathrm{y}$ aprendizaje de los contenidos del curso Lenguaje y Percepción Musical en los estudiantes de Educación Musical del Instituto Pedagógico de Miranda José Manuel Siso Martínez, de la UPEL. Allí se evidenció que por medio de lo conocido se puede asimilar lo desconocido, es decir la conexión de lo autodidacta con lo académico en la enseñanza del lenguaje y la percepción de la música. Esto, gracias a la relación que existía entre los métodos de enseñanza musical con los conocimientos previos de los estudiantes.
Perfeccionar el diseño y continuar con la aplicación consciente de la propuesta derivada de la modalidad sobre la cual se apoya el tipo de investigación debe ser una tarea sostenida, no solo en los cursos de Lenguaje y Percepción Musical sino también en los demás cursos e instituciones que imparten la especialidad de Educación Musical.

\section{REFERENCIAS}

Alfonzo, A. (2003). Estrategias Instruccionales. Caracas: Mimeo Ángeles, O. (2003). Enfoques y Modelos Educativos Centrados en el Aprendizaje: $\quad$ Fundamentos Psicopedagógicos de los Enfoques y Estrategias Centrados en el Aprendizaje en el Nivel de Educación Superior [versión electrónica]. México: SEP. Recuperado de http://ses4.sep.gob.mx/

Anijovich, R. y Mora, S. (2009). Estrategias de enseñanza. Otra mirada al quehacer en el aula. Buenos Aires: Aique Grupo Editor Arias, F. (2006). El proyecto de investigación, introducción a la metodología científica. México: Editorial Episteme

Ausubel, D., Novak, J. y Hanesian H. (1983). Psicología Educativa: Un punto de vista cognoscitivo. $2^{\circ} \mathrm{Ed}$. México: Editorial Trillas

Instituto Emile Jaques Dalcroze. (2010). Créativité. Mouvement. Musiqué. Recuperado de http://dalcroze.eu/

Dávila, C. (2011). Estrategias de evaluación que contribuyen al aprendizaje de instrumentos musicales: 
un estudio de caso. (Tesis de maestría) Universidad San Francisco de Quito, Ecuador. Recuperado de http://repositorio.usfq.edu.ec/bitst ream/23000/924/1/99792.pdf

Díaz, F. y Hernández, G. (2010). Estrategias docentes para un aprendizaje significativo. Una interpretación constructivista. México: Mc Graw Hill

Feo, R. (2010). Orientaciones básicas para el diseño de estrategias didácticas. Tendencias Pedagógicas. Portal de Revistas electrónicas UAM, $16,220-236$

Fundación Carl Orff. (1984). Carl Orff. Recuperado de http://www.orff.de/es/vida.html

Gallegos, F. (2010). Rediseño del Programa de Enseñanza Musical de la Licenciatura en Puericultura de la Universidad Tangamanga, México. (Tesis de maestría), Universidad de Tangamanga, San Luis Potosé, México. Recuperado de http://conocimiento.aliat.edu.mx/ cgi-bin/koha/opacdetail.pl?biblionumber $=25813$

García, Z. (2009) La enseñanza de la música en las universidades de Venezuela. Docencia Universitaria, $X(2), \quad$ Recuperado de http://www.ucv.ve/fileadmin/user

upload/sadpro/Documentos/doce ncia_vol10_n2_2009/4_Zaida_Gar cia.pdf

Gentile, M. (2011). El origen del arte musical. ROSARIARTE.

Recuperado de http://www.rosariarte.com.ar/cont enidos $/$ index.php?nota $=42$

Hernández, G. (1998). Paradigmas en Psicología de la educación. México: Editorial Paidós
Hernández, R., Fernández, C. y Baptista, P. (2010). Metodología de la investigación. México: Mc Graw Hill

Leiva, M. y Mates, E. (2002). La educación musical: algo imprescindible. Filomúsica. Recuperado de http://www.filomusica.com/filo33 /educacion.html

Linares, S. (2005). Programa de curso Lenguaje y Percepción Musical. La Urbina, Caracas: Instituto Pedagógico de Miranda José Manuel Siso Martínez

Linares, S. (2016). Estrategias de enseñanza orientadas a la integración del conocimiento autodidacta $y$ académico en estudiantes de educación musical de UPEL-IPM. (Tesis de Maestría) Universidad Pedagógica Experimental Libertador, Miranda, Venezuela

Rodríguez, S. (2003). Corrientes pedagógico-musicales del siglo XX. Análisis y proyección de las mismas en la educación musical escolar. Aula Abierta. Recuperado de http://www.aulaabierta.org/aulaa bierta2/archivos/primaria/temario $\% 20$ muestra $\% 20$ m $\%$ FAsica $\% 201 \%$ AA.pdf

Sanjosé, V. (2003). Didáctica de la expresión musical para maestros. Valencia, España: Editorial Piles

UPEL. (2016). Manual de trabajos de Grado de Especialización y Maestría y Tesis Doctorales. Caracas, Venezuela: Fundación Editorial de la Universidad Pedagógica Experimental Libertador

Uribe, C. (2010). Compresión Musical: Resultados de la Aplicación de una Propuesta Metodológica en un Curso del 
Programa de la Licenciatura en Música de la Universidad Tecnológica de Pereira. (Tesis de maestría) Universidad Tecnológica de Pereira, Colombia. Recuperado de http://recursosbiblioteca.utp.edu.c o/tesisdigitales/texto/78077U76.p df

Vygotski, L. (1995). Pensamiento y Lenguaje. Buenos Aires: Ediciones Fausto
Yépez, A. (2011). Aproximación teórica a los métodos de enseñanza en la educación musical. (Trabajo de ascenso). Instituto Pedagógico de Miranda José Manuel Siso Martínez, Universidad Pedagogía Experimental Libertador, Caracas, Venezuela 